\title{
Codebook para Beamforming de Ondas Milimétricas em Terminais Móveis
}

\author{
Jonas N. da C. Silva, Lisandro Lovisolo e Michel P. Tcheou
}

Resumo-Este trabalho investiga o projeto de codebooks para conformação de feixes em ondas milimétricas em terminais móveis. Para concentrar a potência na direção desejada, aplicase um vetor de deslocamentos de fase a um arranjo de antena. De forma a realizar a conformação com baixa complexidade, em um terminal móvel, define-se um codebook, do qual escolhese o codeword que define os deslocamentos para conformar o feixe. Neste trabalho, reduz-se a quantidade de elementos de um codebook de muitos elementos, utilizando o algoritmo Kmeans. Os resultados mostram que o método proposto obtém um codebook reduzido com desempenho superior ao indicado em IEEE 802.15.3c em termos da cobertura esférica obtida.

Palavras-Chave-Beamforming, Deslocamento de fase, Cobertura esférica.

Abstract-This paper aims at the design of codebooks for beamforming from mobile terminals for millimeter waves. A phase shift vector is applied to the antenna array to concentrate the power in the desired direction. In order to perform the beamforming with low complexity, in a mobile terminal, a codebook is defined, and one chooses the codeword defining the phase shifts to beamform in the desired direction. In this paper, a smaller codebook is designed from a greater codebook using the K-means algorithm. The results show that the proposed method obtains a codebook with superior performance than the one from in IEEE 802.15.3c regarding spherical coverage.

Keywords-Milimeter wave, Beamforming, Codebook, Phase shifts, Spherical coverage, K-means.

\section{INTRODUÇÃO}

Para compensar a pequena cobertura angular de uma antena em ondas milimétricas, a conformação de feixe (beamforming) é utilizada visando concentrar a energia de transmissão em direções específicas [1], [2], [?]. Para isso, o mesmo sinal é irradiado a partir de diferentes elementos de antena com diferentes fases(e, talvez, amplitudes) para formar um feixe em uma direção específica [2]. Ao vetor de pesos complexos que produzem os deslocamentos de fase atribui-se a insígnia de codeword [3]; e um codebook compreende um conjunto de codewords [4].

Este trabalho discorre sobre o projeto de codebooks cujos codewords permitam cobrir o mais uniformemente a esfera. Isso possibilita escolher o codeword para que o terminal móvel irradie mais na direção de interesse. A utilização de beamforming em ondas milimétricas é de suma importância para formar arranjos com alto ganho e compensar as grandes perdas de propagação devido ao pequeno comprimento de onda [3]. Por exemplo, [5] considera esse cenário empregando um arranjo linear uniforme de antenas (ULA - uniform linear

J. N. da C. Silva, L. Lovisolo e M. P. Tcheou, Faculdade de Engenharia, Programa de Pós-Graduação em Engenharia Eletrônica (PEL), Universidade do Estado do Rio de Janeiro (UERJ), Rio de Janeiro - RJ, e-mail: joneco@gmail.com, lovisolo@eng.uerj.br, mtcheou@eng.uerj.br. array) em um terminal móvel. Considerando o alto custo de implementação, o consumo energético, e a forma da cadeia de radiofrequência ( $R F$ Chain), os terminais móveis dificilmente adotam conformação de feixe híbrida ou totalmente digital em ondas milimétricas, já que isso exige mais de uma cadeia de RF por arranjo de antena [6]. Assim, neste trabalho, um codebook de beamforming analógico é considerado.

Este trabalho visa projetar um codebook contendo $K$ codewords para aplicar aos sinais que alimentam os $L$ elementos de antena de uma ULA em um terminal móvel. Parte-se de um codebook contendo uma grande quantidade de codewords e selecionam-se as $K$ que provêm a maior cobertura da esfera unitária. O codebook obtido para $K=16$ e $L=4$ obteve um desempenho superior em termos de ganho médio na esfera unitária comparativamente ao codebook definido por IEEE 802.15.3 [7].

\section{A. Organização}

Na Seção II, formula-se o problema de projeto de conformação de feixes e um método para a avaliação de desempenho de codebooks conformadores. Na Seção III, apresenta-se um método para seleção das melhores codewords de um codebook, utilizando o critério de cobertura da esfera unitária. Na Seção IV, mostram-se resultados de simulação do codebook obtidos com os algoritmos propostos, além da análise comparativa de desempenho relativamente ao codebook de referência presente na normativa IEEE 802.15.3c. Na Seção V, apresentam-se as conclusões obtidas neste trabalho.

\section{B. Notação}

Letra maiúscula e em negrito (X) representa uma matriz, letra minúscula e em negrito $(\mathbf{x})$ representa um vetor, $\arg (X) \in[0,2 \pi)$ denota a fase de um número complexo, $\bmod (x, n)$ se refere ao resto da divisão de $x$ por $n,\|\mathbf{x}\|$ é a norma (quadrática) do vetor $\mathrm{x}$, e $x \sim \mathcal{C N}(\mu, \tau)$ indica que $x$ é uma variável aleatória gaussiana complexa de média $\mu$ e variância $\tau$.

\section{CONFORMAÇÃO DE FEIXES}

O sistema de coordenadas utilizado neste artigo é mostrado na Figura II. O terminal é posicionado na origem, $\phi$ é o ângulo de azimute e $\theta$ é o ângulo de elevação. Considera-se o campo eletromagnético na região de campo distante (Fraunhofer), no qual as ondas eletromagnéticas são consideradas planas [1]. O campo elétrico é um vetor tal que sua componente radial, $e^{R}$, é nula ou muito pequena em relação às componentes $e^{\Theta}$ e $e^{\Phi}$ [1]. 


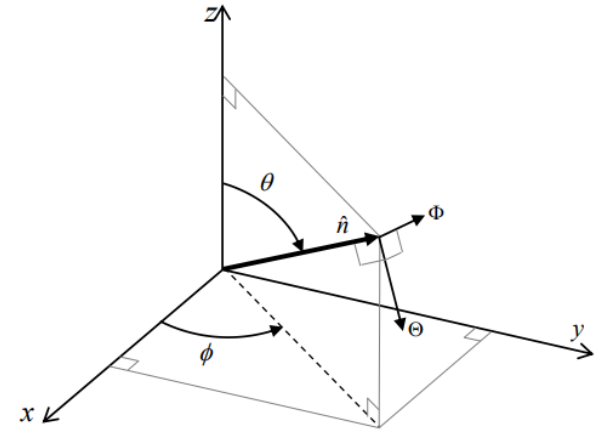

Fig. 1. Sistema de coordenadas empregado, destacam-se os ângulos de azimute $\phi$ e de elevação $\theta$.

Assumindo uma ULA com $L$ elementos de antena indexados por $\ell=1,2, \ldots, L$, as componentes de azimute e elevação $(\theta, \phi)$ do campo elétrico irradiado pelo l-ésimo elemento da ULA são $e_{\ell}^{\Phi}(\theta, \phi)$ e $e_{\ell}^{\Theta}(\theta, \phi)$, respectivamente. A partir das quais, definem-se os vetores

$$
\begin{array}{r}
\mathbf{e}^{\Theta}(\theta, \phi) \triangleq\left[e_{1}^{\Theta}(\theta, \phi), e_{2}^{\Theta}(\theta, \phi), \ldots, e_{L}^{\Theta}(\theta, \phi)\right] \\
\mathbf{e}^{\Phi}(\theta, \phi) \triangleq\left[e_{1}^{\Phi}(\theta, \phi), e_{2}^{\Phi}(\theta, \phi), \ldots, e_{L}^{\Phi}(\theta, \phi)\right]
\end{array}
$$

Um codebook é definido por

$$
\mathbf{W}_{c} \triangleq\left\{\mathbf{w}_{1}, \mathbf{w}_{2}, \ldots, \mathbf{w}_{K}\right\},
$$

onde $\mathbf{w}_{i}, i=1 \ldots K$, é um vetor de pesos complexos que são aplicados aos elementos de antena para realização do beamforming. $\mathrm{O}$ codebook $\mathbf{W}_{c}$ contém $K$ elementos de norma unitária, isto é, $\left\|\mathbf{w}_{k}\right\|=1$.

De acordo com o princípio da superposição, as componentes dos campos elétricos $\mathbf{e}^{\Theta}(\theta, \phi)$ e $\mathbf{e}^{\Phi}(\theta, \phi)$ com a multiplicação pelo vetor de pesos de beamforming $\mathbf{w}$ são

$$
\begin{aligned}
& \varepsilon_{\mathbf{w}(\theta, \phi)}^{\Theta}=\sum_{\ell=1}^{L} w_{\ell}^{*} e_{\ell}^{\Theta}(\theta, \phi)=\mathbf{w}^{H} \mathbf{e}^{\Theta}(\theta, \phi) \\
& \varepsilon_{\mathbf{w}(\theta, \phi)}^{\Phi}=\sum_{\ell=1}^{L} w_{\ell}^{*} e_{\ell}^{\Phi}(\theta, \phi)=\mathbf{w}^{H} \mathbf{e}^{\Phi}(\theta, \phi)
\end{aligned}
$$

Com a resposta complexa do campo elétrico, podemos definir o ganho de beamforming. Tal ganho é direcional e dependente da resposta de campo eletromagnético (M)

$$
\begin{aligned}
G_{\mathbf{w}} & (\theta, \phi)=\frac{4 \pi}{\|\mathbf{w}\|^{2}} \cdot \frac{1}{2 \eta_{0}}\left(\left|\varepsilon_{\mathbf{w}}^{\Theta}(\theta, \phi)\right|^{2}+\left|\varepsilon_{\mathbf{w}}^{\Phi}(\theta, \phi)\right|^{2}\right) \\
= & \frac{2 \pi}{\eta_{0}}\left(\mathbf{w}^{H}\left(\mathbf{e}^{\Theta}(\theta, \phi) \mathbf{e}^{\Theta}(\theta, \phi)^{H}+\mathbf{e}^{\Phi}(\theta, \phi) \mathbf{e}^{\Phi}(\theta, \phi)^{H}\right) \mathbf{w}\right) \\
= & \frac{2 \pi}{\eta_{0}} \mathbf{w}^{H} \mathbf{M}(\theta, \phi) \mathbf{w}
\end{aligned}
$$

onde $\eta_{0} \approx 377 \Omega$ é a impedância do espaço livre. A matriz de resposta do campo eletromagnético $\mathbf{M}(\theta, \phi)$ é

$$
\mathbf{M}(\theta, \phi) \triangleq \mathbf{e}^{\Theta}(\theta, \phi) \mathbf{e}^{\Theta}(\theta, \phi)^{H}+\mathbf{e}^{\Phi}(\theta, \phi) \mathbf{e}^{\Phi}(\theta, \phi)^{H}
$$

A expressão $\mathbf{w}^{H} \mathbf{M}(\theta, \phi) \mathbf{w}$ pondera a matriz de resposta do campo elétrico pelos pesos em cada componente angular.
Ao projetar o codebook, pretendemos que

$$
\begin{array}{r}
\mathbf{W}_{\mathbf{c}}{ }^{*}=\max _{\mathbf{W}_{\mathbf{c}}} U\left(S\left(\mathbf{W}_{\mathbf{c}}, \theta, \phi\right)\right) \text { onde } \\
S\left(\mathbf{W}_{\mathbf{c}}, \theta, \phi\right)=\frac{2 \pi}{\eta_{0}} \max _{\mathbf{w}_{\mathbf{k}} \in W_{c}} \mathbf{w}_{k}^{H} \mathbf{M}(\theta, \phi) \mathbf{w}_{k},
\end{array}
$$

A função de utilidade $U(\cdot)$ é definida como o ganho médio sob toda a esfera unitária, a esfera unitária corresponde a uma esfera teórica cuja posição geométrica é dada pela equação: $x^{2}+y^{2}+z^{2}=1$. Ou então a função utilidade pode ser um percentil do ganho sob a esfera unitária [4]. Neste trabalho, utilizaremos o percentil 50 da cobertura da esfera unitária para avaliação do terminal móvel ou UE (User Equipment), seguindo o critério definido em [8]. A partir da função utilidade selecionam-se os melhores codewords para formar o codebook ótimo $\left(\mathbf{W}_{c}^{*}\right)$. A equação (11) define o fator de desempenho do codebook $S\left(W_{c}, \theta, \phi\right)$, a cobertura da esfera por um codebook, o ganho de beamforming máximo obtido com as codewords na direção dada. Ele pode ser usado para identificar "buracos" de cobertura ou grandes sobreposições de feixes gerados.

Assumimos que apenas um diagrama de radiação é ativado em cada instante de tempo, logo o fator de desempenho equivale ao máximo de cada um dos diagramas individuais do codebook [4]. [4] difere na resolução do deslocamento de fase do codebook gerado, que não está pré-limitada a uma quantidade específica de bits, mas sim, à frequência espacial presente no modelo de antena utilizado, ou seja aos angulos $\Theta$ calculados em 20 .

A Figura 2(a) mostra os feixes gerados por um codebook e a Figura 2(b) exemplifica o fator de cobertura de um codebook genérico com 5 codewords, aplicado em uma ULA com 4 elementos.

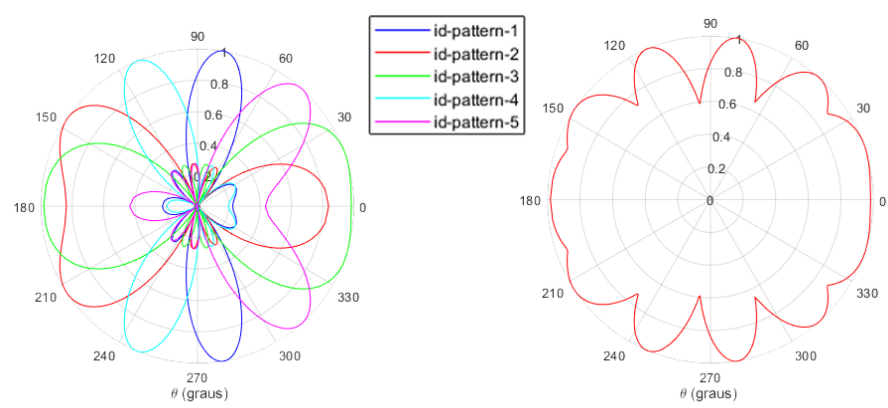

(a) Feixes gerados pelo CodeBook

(b) Máximo Ganho entre os feixes (S)

Fig. 2. Feixes gerados por um codebook e o fator de cobertura resultante, ambos no diagrama azimutal.

\section{A. Conformação de feixes simples}

Dada a matriz de resposta de campo elétrico na direção $(\theta, \phi), \mathbf{M}(\theta, \phi)$, ou a soma da resposta do campo elétrico em um conjunto de direções $\mathbf{M}=\sum_{(\theta, \phi) \in A} \mathbf{M}(\theta, \phi)$, deseja-se obter o vetor que maximiza o ganho de beamforming $\mathbf{w}^{H} \mathbf{M w}$.

No projeto do codebook, as codewords estão sujeitas a restrições de potência por elemento. Tal restrição pode ser 
formulada como

$$
\begin{gathered}
\mathbf{M}^{*}=\arg \left(\max _{\mathbf{w}:\left|w_{i}\right| \leq \frac{1}{\sqrt{L}} \forall i} B_{1}(\mathbf{M})\right), \\
\text { com } B_{1}(\mathbf{M}) \triangleq \mathbf{w}^{* H} \mathbf{M} \mathbf{w}^{*}
\end{gathered}
$$

o $\mathbf{w}^{*}$ (peso ótimo) deve utilizar completamente a potência, ou seja, $\left|w_{i}\right|=\frac{1}{\sqrt{L}}[9]$. Se $\operatorname{rank}(\mathbf{M})=1, \mathbf{M}=\mathbf{m m}^{H}$, então a solução ótima é $w_{i}^{*}=\frac{m_{i}}{\left|m_{i}\right| \sqrt{L}}$ e $B_{1}=\frac{1}{L}\left(\sum_{i}\left|m_{i}\right|\right)^{2}$.

Utilizaremos um algoritmo iterativo de descida coordenada com restrição de potência por elemento de antena. Essa restrição por elemento de antena implica que cada elemento de antena possui um amplificador de potência independente, sendo limitada individualmente pela linearidade desses amplificadores [10]. O Algoritmo 1 apresenta a solução. Apesar da garantia de convergência para uma solução estacionária localmente ótima [9], [11], a solução pode não corresponder ao ótimo global.

Algoritmo 1: Algoritmo iterativo de descida coordenada com restrição de potência por elemento de antena

1) Inicializar $i \leftarrow 1 \quad$ e $\quad \mathbf{w} \sim \mathcal{C N}(0, I)$

2) Atualizar $w_{i}$ como:

$$
w_{i} \leftarrow \frac{1}{\sqrt{L}} \exp \left(j \arg \left(\sum_{k \neq i} M_{i k} w_{k}\right)\right)
$$

3) Verificar a convergência do ganho de beamforming de acordo com a Equação (12); Caso afirmativo, parar; caso contrario, $i \leftarrow \bmod (i, L)$ e retornar ao Passo 2 .

\section{B. Limite superior de feixe de antena composto}

O limite superior (UpperBound) é derivado da resposta do campo eletromagnético $\left(e_{\Theta}\right.$ e $\left.e_{\Phi}\right)$ e é independente do tamanho de codebook $(K)$. Este parâmetro será a base de avaliação de desempenho dos codebooks presentes neste trabalho.

O problema de limite superior sobre toda a esfera $(\theta, \phi) \in$ $\left[0^{\circ}, 180^{\circ}\right] \times\left[0^{\circ}, 360^{\circ}\right)$ pode ser formulado como:

$$
\begin{array}{r}
\bar{S}(\theta, \phi) \triangleq \frac{2 \pi}{\eta_{0}} \max _{\|\mathbf{w}\| \leq 1} \mathbf{w}^{H} \mathbf{M}(\theta, \phi) \mathbf{w} \\
\bar{S}(\theta, \phi) \triangleq \frac{2 \pi}{\eta_{0}} \lambda_{\max }(\mathbf{M}(\theta, \phi))
\end{array}
$$

onde $\lambda_{\max }(X)$ representa o máximo autovalor de $\mathrm{X}$. O limite superior só será atingido por um codebook que consiste no máximo autovetor de $\mathbf{M}(\theta, \phi)$ para cada direção. Dessa maneira, seria necessário remover a limitação de tamanho do codebook, o limite de potência por elemento e o limite de fase discreta para construir um codebook que consiga atingir esse limite superior.

O limite superior de um codebook é simplesmente a junção dos codewords equivalentes aos valores máximos de limite superior de cada um dos feixes. Simplesmente, comparamos o valor de todos os feixes dos codewords individualmente em cada direção e selecionamos o maior ganho dentre eles para formar o diagrama composto.

\section{CONSTRUÇÃO DO codebook PROPOSTO}

K-means é um processo de agrupamento de dados não supervisionado que particiona $n$ observações/dados em $K$ grupos [12]. Utilizaremos o algoritmo K-means para selecionar codewords para formar um codebook. O número $K$ é o tamanho do codebook, ou seja, a quantidade de codewords que irão realizar as diferentes conformações de feixe. O parâmetro utilizado para agrupamento será o limite superior indicado na Equação (15).

Primeiramente, são gerados $K$ centróides aleatórios, e em seguida, aloca-se cada pattern (diagrama de radiação do efeito de cada codeword na antena) ao centróide mais próximo (de menor distância euclidiana). Segundo, deve-se criar uma nova posição para o centróide, que será a média das posições dos patterns pertencentes a este centróide. Em seguida, faremos novamente a alocação dos patterns para o centróide mais próximo. O processo é refeito até a alocação ótima dos centróides ser atingida. Vale ressaltar que a cada vez que rodarmos o algoritmo, inicialmente teremos uma diferença nos centroides, uma vez que a escolha inicial é um processo aleatório. Logo, há uma possibilidade de atingirmos uma solução ótima não global.

Neste trabalho, utilizamos o Algoritmo 1 para realizar um codebook com $K$ demasiadamente grande, compreendendo toda a resolução/frequência espacial de $\Theta$ no modelo de antena utilizado. O limite superior desse codebook de tamanho grande codebook será muito próximo ao de uma antena omnidirecional. Então utilizaremos o Algoritmo 2, que emprega K-means para selecionarmos desse codebook grande, um codebook ótimo com $K=16$. A Figura 5 mostra o codebook gerado pelo algoritmo de seleção de codewords proposto.

Algoritmo 2: Algoritmo para seleção de codebook usando $\mathrm{K}$-means

1) Dado um conjunto de $\mathrm{N}$ codewords $\left\{\mathbf{w}_{\mathbf{1}}, \mathbf{w}_{\mathbf{2}}, \ldots, \mathbf{w}_{\mathbf{N}}\right\}$ e desejamos selecionar $\mathrm{K}$ codewords para formar um codebook ótimo e a direção de máximo ganho relacionada a cada codeword .

$$
\mathcal{D}_{n}=\left\{(\theta, \phi) \mid \arg \left(\max \left(\mathbf{w}_{n}^{H} \mathbf{M}(\theta, \phi) \mathbf{w}_{n}\right)\right)\right\}
$$

onde, $1 \leq n \leq N$.

2) Realizar o processo de particionamento K-means para identificar $\mathrm{K}$ centróides dentre todas as direções calculadas no processo anterior $C_{k}(\theta, \phi)$.

3) Escolher as $\mathrm{K}$ melhores codewords de acordo com a menor distância euclidiana a cada um dos centróides $W^{*}=\left[W_{1}^{*}, W_{2}^{*}, \ldots, W_{K}^{*}\right]$

$$
w_{k}^{*}=\min \left\|C_{k}(\theta, \phi)-\mathcal{D}_{n}(\theta, \phi)\right\|,
$$

onde, $1 \leq k \leq K, 1 \leq n \leq N$.

\section{Resultados e Simulações}

\section{A. Codebook referência IEEE 802.15.3c}

O problema deste trabalho está em prover um Codebook de tamanho $K=16$ e composto por $L=4$ antenas que seja mais eficiente que o codebook proposto na normativa IEEE 
802.15.3c [7]. Foi escolhido o tamanho do codebook como $\mathrm{K}=16$, pois com esse valor ocorreram poucas sobreposições de feixes com uma grande cobertura da esfera unitária, como mostrado em [4]. A normativa 802.15 .3 c propõe um codebook para ondas milimétricas, mais precisamente em $60 \mathrm{GHz}$ [13]. Os patterns produzidos por esse codebook estão ilustrados na Figura 3. Os pesos desse Codebook são formulados como:

$$
w(\ell, k)=\frac{1}{\sqrt{\ell}} \exp \left(j \frac{2 \pi}{4}\left\lfloor\bmod \left(\frac{(\ell+(K / 2), K}{K / 4}\right)\right\rfloor\right)
$$

\section{CodeBook - IEEE 802.15.3c $K=16 \mathrm{~L}=4$}

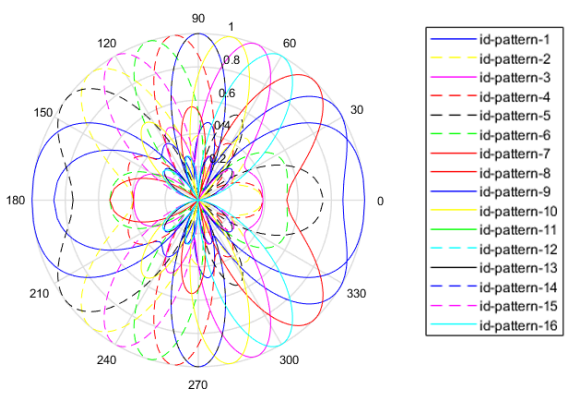

Fig. 3. Diagrama azimuthal para o codebook presente na normativa IEEE 802.15.3c $K=16$ e $L=4$.

onde, $1 \leq \ell \leq L, 1 \leq k \leq K$. A função $\lfloor\mathrm{x}\rfloor$ corresponde ao maior inteiro menor que $\mathrm{x}, K$ é o tamanho do codebook e $L$ é a quantidade de antenas do arranjo.

\section{B. Modelo de Antena Utilizado no Codebook proposto}

Nesta seção, iremos apresentar o modelo de antena que utilizaremos para o projeto de codebook. Utilizaremos um modelo ULA (Uniform Linear Array), onde todos os elementos presentes no conjunto de antenas são iguais. Os dados de campo elétrico são gerados para as direções angulares uniformemente. Como os elementos da ULA estão dispostos apenas em uma direção linear, assumiremos um arranjo de antena de genérico de tamanho $1 \times L$. Utilizaremos um modelo direcional de antena no qual o diagrama de radiação é dado por:

$$
p(\theta)=\sin ^{q}(\theta)
$$

O parâmetro $q$ é um parâmetro de diretividade que permite "achatar" o lóbulo de radiação da antena. Esse efeito pode ser visto na Figura 4.

O campo eletromagnético para as direções angulares é uniformemente distribuído sobre a frequência espacial, ou seja $\theta=\arccos (x), x=[-1,-(a-1) / a, \ldots,(a-1) / a, 1]$. Assumindo um ULA $1 \times L, a=30 L$ [4]. O campo eletromagnético em $\theta$ será:

$$
e^{\Theta}(\theta)=\sqrt{p(\theta)} \exp \left(j \frac{2 \pi \cos (\theta)}{d}[0,1, \ldots, L-1]^{T}\right),
$$

e $\mathbf{e}^{\Phi}(\theta)=0$, uma vez que em uma ULA não há radiação perpendicular ao conjunto.

O codebook proposto obtido por meio do método descrito na Seção III,está ilustrado na Figura 5.
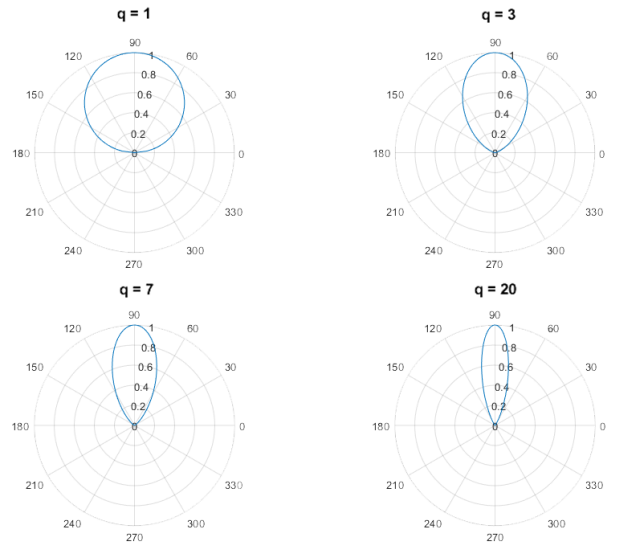

Fig. 4. Impacto do parâmetro $q$ no diagrama azimuthal do modelo de antena proposto.

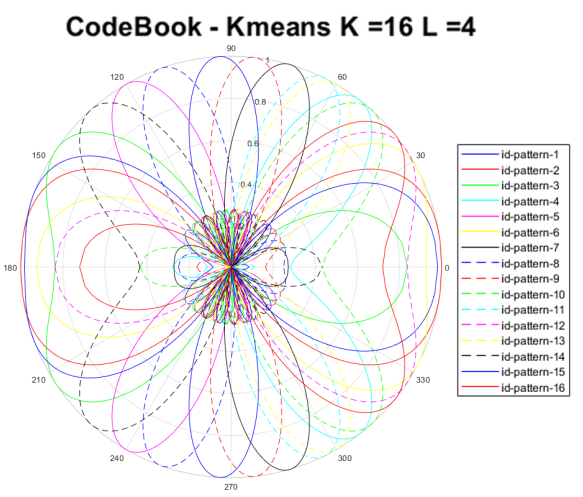

Fig. 5. Diagrama azimuthal para o codebook proposto $K=16$ e $L=4$.

\section{Comparativo entre o Codebook Proposto e o Codebook Referência}

A CDF da cobertura do codebook sobre a esfera unitária é representada na Figura 7. Observa-se que o codebook proposto atinge níveis de cobertura da esfera unitária mais rapidamente. $\mathrm{O}$ percentil $\mathrm{X}$ (representado no eixo $\mathrm{Y}$ ) indica que $\mathrm{X} \%$ dos pontos presentes na esfera unitária teórica estão cobertos por uma determinada intensidade. Pela CDF, podemos ver que o codebook proposto atinge uma cobertura da esfera unitária mais eficiente. A Figura 6 mostra o limite superior dos codebooks, com base nela, percebe-se que há uma melhor cobertura da esfera teórica e uma menor diferença entre o valor máximo e o mínimo do diagrama de radiação. A Figura 6(a) representa o limite superior do codebook referência IEEE 802.15.3c e o codebook obtido neste trabalho. A

A Tabela I mostra as métricas da simulação realizada no codebook referência e no codebook obtido neste trabalho, em que $U$ medio é a função utilidade que representa ganho médio em toda esfera unitária e $U 50$ é o percentil 50 do ganho em toda esfera unitária como descrito na Seção II.

$\mathrm{Na}$ Tabela I, os limites superiores ilustrados na Figura 6 e o gráfico de CDF na Figura 7, concluímos que o codebook um desempenho superior ao codebook do IEEE 802.15.3c. Ele possui uma menor variação entre o ganho máximo e mínimo 
TABELA II

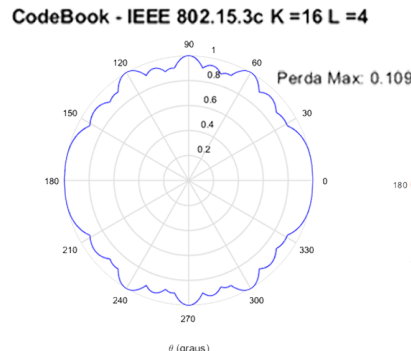

(a)

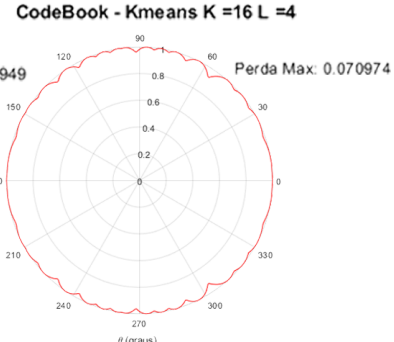

(b)
Fig. 6. Codebook obtido neste trabalho.

TABELA I

COMPARAÇÃO ENTRE OS codebooks DE IEEE 802.15.3C E O OBTIDO NESTE TRABALHO.

\begin{tabular}{|c|c|c|c|}
\hline codebook & Perda Max & Umedio(dB) & U50 (dB) \\
\hline IEEE 802.15.3c & 0,109 & $-0,510$ & $-0,560$ \\
\hline Obtido & 0,074 & $-0,099$ & $-0,050$ \\
\hline
\end{tabular}

dos feixes presentes no codebook e também possui uma melhor cobertura da esfera unitária para o terminal.

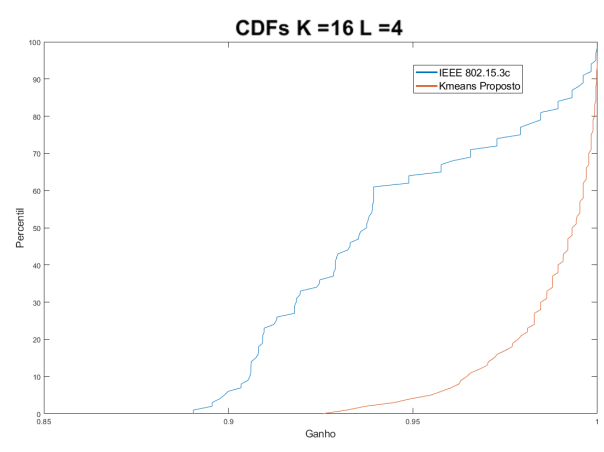

Fig. 7. CDF da cobertura da esfera unitária do Codebook referência e do codebook obtido neste trabalho.

\section{Conclusões}

Neste trabalho, foram desenvolvidas técnicas para o projeto de codebooks para conformação de feixe em ondas milimétricas em terminais móveis. De forma a realizar a conformação com baixa complexidade, em um terminal móvel, define-se um codebook, do qual seleciona-se o codeword que define os deslocamentos para conformar o feixe. Neste trabalho, considerou-se como critério de desempenho a cobertura esférica que o codebook provê com o arranjo de antenas do terminal móvel. A Tabela II contém as codewords utilizadas no codebook obtido neste trabalho, porém por motivos de formatação ela está apresentada de forma transposta. Propomos o uso de um algoritmo iterativo para projeto de um codebook inicialmente grande e, então, empregamos o algoritmo $K$-means para selecionar um codebook ótimo com menos elementos. Utilizando essa proposta, projetaram-se codebooks com o método desenvolvido com desempenho superior ao especificado em no IEEE 802.15.3c.
COMPARAÇÃO ENTRE OS codebooks DE IEEE 802.15.3C E O OBTIDO NESTE TRABALHO.

\begin{tabular}{|c|c|c|c|}
\hline$-0,47+0,171 i$ & 0,5 & $-0,47-0,171 i$ & $0,383+0,321 i$ \\
\hline$-0,433-0,25 i$ & 0,5 & $-0,433+0,25 i$ & $0,25-0,433 i$ \\
\hline$-0,492-0,0868 i$ & 0,5 & $-0,492+0,0868 i$ & $0,47-0,171 i$ \\
\hline$-0,25-0,433 i$ & 0,5 & $-0,25+0,433 i$ & $-0,25-0,433 i$ \\
\hline $0,171-0,47 i$ & 0,5 & $0,171+0,47 i$ & $-0,383+0,321 i$ \\
\hline 0,5 & $0,433-0,25 i$ & $0,25-0,433 i$ & $-1,34 e-12-0,5 i$ \\
\hline $0,0868+0,492 i$ & 0,5 & $0,0868-0,492 i$ & $-0,47-0,171 i$ \\
\hline$-0,321+0,383 i$ & 0,5 & $-0,321-0,383 i$ & $-0,0868+0,492 i$ \\
\hline $0,433-0,25 i$ & $-0,383-0,321 i$ & $-0,171+0,47 i$ & 0,5 \\
\hline$-0,47+0,171 i$ & 0,5 & $-0,47-0,171 i$ & $0,383+0,321 i$ \\
\hline 0,5 & $0,433+0,25 i$ & $0,25+0,433 i$ & $1,41 e-12+0,5 i$ \\
\hline $0,321-0,383 i$ & 0,5 & $0,321+0,383 i$ & $-0,0868+0,492 i$ \\
\hline $0,5+7,68 e-13 i$ & $0,5+5,12 e-13 i$ & $0,5+2,56 e-13 i$ & 0,5 \\
\hline $0,321+0,383 i$ & 0,5 & $0,321-0,383 i$ & $-0,0868-0,492 i$ \\
\hline 0,5 & $0,433-0,25 i$ & $0,25-0,433 i$ & $-1,83 e-13-0,5 i$ \\
\hline $0,383+0,321 i$ & $-0,47-0,171 i$ & 0,5 & $-0,47+0,171 i$ \\
\hline
\end{tabular}

\section{Agradecimentos}

O presente trabalho foi realizado com apoio da Coordenação de Aperfeiçoamento de Pessoal de Nível Superior - Brasil (CAPES) - Código de Financiamento 001.

\section{REFERÊNCIAS}

[1] Constantine A Balanis. Antenna theory: analysis and design. John wiley \& sons, 2015.

[2] Kristian Zarb-Adami, A Faulkner, JG Bij de Vaate, GW Kant, and $\mathrm{P}$ Picard. Beamforming techniques for large-n aperture arrays. In 2010 IEEE International Symposium on Phased Array Systems and Technology, pages 883-890. IEEE, 2010.

[3] Wonbin Hong, Kwang-Hyun Baek, Youngju Lee, Yoongeon Kim, and Seung-Tae Ko. Study and prototyping of practically large-scale mmwave antenna systems for $5 \mathrm{~g}$ cellular devices. IEEE Communications Magazine, 52(9):63-69, 2014.

[4] Jianhua Mo, Boon Loong Ng, Sanghyun Chang, Pengda Huang, Mandar N Kulkarni, Ahmad AlAmmouri, Jianzhong Charlie Zhang, Jeongheum Lee, and Won-Joon Choi. Beam codebook design for $5 \mathrm{~g}$ mmwave terminals. IEEE Access, 7:98387-98404, 2019.

[5] Mingming Cai, J Nicholas Laneman, and Bertrand Hochwald. Beamforming codebook compensation for beam squint with channel capacity constraint. In 2017 IEEE International Symposium on Information Theory (ISIT), pages 76-80. IEEE, 2017.

[6] Kenichi Kagoshima. Pattern control antennas for wireless access systems. In IEEE Antennas and Propagation Society International Symposium. Transmitting Waves of Progress to the Next Millennium. 2000 Digest. Held in conjunction with: USNC/URSI National Radio Science Meeting (C, volume 2, pages 574-577. IEEE, 2000.

[7] Zou Weixia, Guo Chao, Du Guanglong, Wang Zhenyu, and Gao Ying A new codebook design scheme for fast beam searching in millimeterwave communications. China Communications, 11(6):12-22, 2014.

[8] Evolved Universal Terrestrial Radio Access. User equipment (ue) radio transmission and reception (release 10)," 3gpp ts 36.101. V10, 2, 2011.

[9] Zhouyue Pi. Optimal transmitter beamforming with per-antenna power constraints. In 2012 IEEE International Conference on Communications (ICC), pages 3779-3784. IEEE, 2012.

[10] Wei Yu and Tian Lan. Transmitter optimization for the multi-antenna downlink with per-antenna power constraints. IEEE Transactions on signal processing, 55(6):2646-2660, 2007.

[11] Wei Yu, Wonjong Rhee, Stephen Boyd, and John M Cioffi. Iterative water-filling for gaussian vector multiple-access channels. IEEE Transactions on Information Theory, 50(1):145-152, 2004.

[12] James MacQueen et al. Some methods for classification and analysis of multivariate observations. In Proceedings of the fifth Berkeley symposium on mathematical statistics and probability, volume 1, pages 281-297. Oakland, CA, USA, 1967.

[13] Tuncer Baykas, Chin-Sean Sum, Zhou Lan, Junyi Wang, M Azizur Rahman, Hiroshi Harada, and Shuzo Kato. Ieee 802.15. 3c: The first ieee wireless standard for data rates over $1 \mathrm{gb} / \mathrm{s}$. IEEE Communications Magazine, 49(7):114-121, 2011. 